\title{
An Energy Efficient Architecture for Furnace Monitor and Control in Foundry Based on Industry 4.0 Using IoT
}

\author{
M. Dinesh $\mathbb{D}^{1},{ }^{1}$ C Arvind $\mathbb{D},{ }^{2}$ S.S Sreeja Mole, ${ }^{3}$ C.S. Subash Kumar, ${ }^{4}$ P. Chandra Sekar, ${ }^{5}$ \\ K. Somasundaram $\mathbb{D D}^{6}{ }^{6}$ K. Srihari, ${ }^{7}$ S. Chandragandhi, ${ }^{8}$ \\ and Venkatesa Prabhu Sundramurthy $\mathbb{D}^{9}$ \\ ${ }^{1}$ Department of Electronics and Communication Engineering, Shri Angalamman College of Engineering and Technology, \\ Trichy, India \\ ${ }^{2}$ Department of Electronics and Communication Engineering, Karpagam College of Engineering, Coimbatore 641032, India \\ ${ }^{3}$ Department of Electronics and Communication Engineering, Christu Jyothi Institute of Technology and Science, Yeswanthapur, \\ Janagon 506167, India \\ ${ }^{4}$ Department of Electrical and Electronics Engineering, PSG Institute of Technology and Applied Research, \\ Coimbatore 641032, India \\ ${ }^{5}$ Department of ECE, Siddartha Institute of Science and Technology, Puttur, Andhra Pradesh, India \\ ${ }^{6}$ Institute of Information Technology, Saveetha School of Engineering, SIMATS, Chennai, India \\ ${ }^{7}$ Department of Computer Science and Engineering, SNS College of Technology, Coimbatore, India \\ ${ }^{8}$ Department of Computer Science and Engineering, JCT College of Engineering and Technology, Coimbatore, India \\ ${ }^{9}$ Center of Excellence for Bioprocess and Biotechnology, Department of Chemical Engineering, \\ College of Biological and Chemical Engineering, Addis Ababa Science and Technology University, Addis Ababa, Ethiopia
}

Correspondence should be addressed to Venkatesa Prabhu Sundramurthy; venkatesa.prabhu@aastu.edu.et

Received 13 October 2021; Revised 22 November 2021; Accepted 8 December 2021; Published 22 January 2022

Academic Editor: M Pallikonda Rajasekaran

Copyright (C) 2022 M. Dinesh et al. This is an open access article distributed under the Creative Commons Attribution License, which permits unrestricted use, distribution, and reproduction in any medium, provided the original work is properly cited.

\begin{abstract}
The global standards in the field of industrial automation are maintained in industries by completely digitizing their manufacturing process with industry 4.0 standard. Internet of Things (IoT) enables the conservation of cultural heritage with proper assistance on data management on the data collected from the sensors. However, energy efficient conservation is required to monitor the IoT sensors in order to deal with building a better infrastructure. In this paper, we develop a bio-inspired algorithm which can automate the entire furnace monitoring and controlling system in order to eliminate the human intervention involved in the physical process. The algorithm is blended as a web-based remote application for the better control of the tasks involved, energy utilized, and its subsequent log-report maintenance. The entire system employs Wi-Fi communication for data transfer from device to cloud where the stored data including temperature log, forth coming schedule, and process graphic are maintained by the proposed algorithm to predict the machine failure at an earlier stage. The real-time prototype system is supported by a heat treatment process that is completely automated using IoT to monitor and maintain the temperature during the production of metal casting process.
\end{abstract}

\section{Introduction}

In automobile sector, the quality and quantity production of vehicle accessories including gears, bearings, brake disk, piston, and brake lever and cam shaft should meet the everlasting demand in the market. Considering its contribution to the economic growth, the intricacy involved in the manufacturing and its assembling process has a set of prescribed particulars and guidelines to be strictly followed based on Industry 4.0 [1]. The vehicle organizations will progressively focus on quality building in the manufacturing process by employing an optimized architecture [2] in order 
to meet the industry standards and to provide quality product to its customers. The automation of the entire process with perfection is desired in order to avoid manual intervention [3].

In order to make the manufactured product more durable and light weight, the heat treat process (HTP) is used. The HTP is applicable for molding metals such as SG iron, aluminum, and steel. The resulting model is initially maintained at a very high temperature for a fixed duration. Later the metal casting is allowed to cool at a sufficiently low temperature in order to vary the meteorology structure without changing its shape. The entire process makes the metal parts harder, stronger, and more resistant to any undesired external impact. The heating process is carried out such that the temperature is maintained uniform at the top, bottom, left, and right side of the metal casting in order to make it stronger.

The steps involved in HTP are annealing, stress reliving, and normalizing. The temperature at which the heat treatment should be performed depends on the mechanical properties of the metal used for molding process. Ahead of the heat treatment, the casting part called cementite and pearlite have a gap, as shown in Figure 1. After the HTP, the cementite and pearlite molecular structure are joined together making the metal more durable. IoT sensors play a major role in finding the temperature during this process. Energy efficient conservation is required to monitor the IoT sensors in order to deal with building a better infrastructure.

The main contribution of the paper involves the following.

A bio-inspired algorithm can automate the furnace monitoring and controlling to eliminate the human intervention in the physical process.

The algorithm is combined with a web-based remote application for the better control of the tasks involved, energy utilized, and its subsequent log-report maintenance.

\section{Automation of Heat Treatment Process}

In underdeveloped or developing countries, manual labor is employed to perform heat treatment of metal casting. The furnace used for casting causes a pungent smoke and the hot ambience is hazardous to human beings. In some cases, the HTP is carried out continuously for two to three days during which the furnace will maintain an ambient temperature of $160^{\circ} \mathrm{C}$. The major limitation of HTP is the health hazard; it directs to the human operators involved in the process. However, the overall hazardous procedure has to be followed to produce high-quality accessories for automobiles [4]. A decade back the furnace automation was supported by PLC-based systems, but they could hardly work after a year or so due its feeble resistance to the high-temperature environment. In the literature, various optimization algorithms are suggested for industrial engineering and automation problems.

2.1. Importance of Heat Treatment in Automobile Industry. Any ambiguity of the internal properties of a metal during melting such as disorientation of microstructure shown in

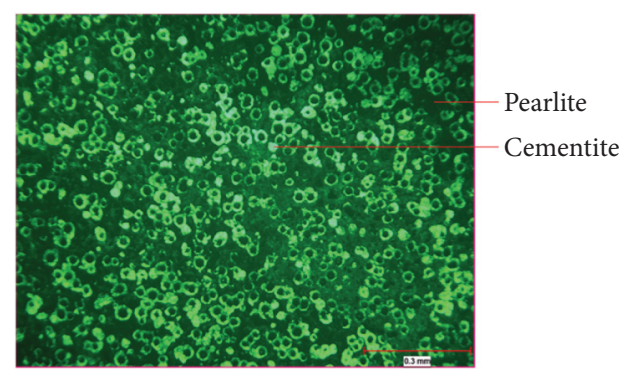

Figure 1: Before heat treatment microstructure.

Figure 1 can be rectified by the heat treatment process. The molecules in a metal are oriented for proper bonding by maintaining it at a constant temperature during the HTP. An appropriate heat treatment can form covalent bond among the molecules (pearlite and cementite) to improve the hardness and strength of the metal. The microstructure bonding after heat treatment is shown in Figure 2. The undesired temperature variation in metal casting (difference in upper and lower section) is shown in Figure 3.

\section{Background: Major Issue in Heat Treatment Process}

3.1. Pollution Area and People Management. To check the regular workflow of heat treatment, there is a particular time and temperature to maintain every casting part based on this instruction. This is given by the manager, the operator has to work on, and the supervisor needs to verify the process. At each interval, the operator has to monitor and record the temperature. Moreover, the operator should be trained; the temperature and time setting will be handled by the heattreatment operator. The furnace operation runs nearly 1 to 2 days; the operator will be changing at shift bases. At the change over time and break time, the operator will not be there at field area if the temperature and time setting should be done right. If any problem occurs at the temperature and time setting, the product will be affected; the affected product will complicate to find it. Manual instruction process flow is shown in Figure 4.

3.2. Quality Checking Process. Over all the companies and organization with respect to quality, the quality inspection system will be checked under customer specification requirement (CSR); CSR will vary for every product. In the quality system, each and every product quantity is not included for quality check. The quality standard is 1 to 10 pieces which will be going for quality inspection in a bundle or batch. Some of the quality-checking inspectors find shortcuts to go fast. Visual inspection and dimension inspections are automobiles major parts.

3.3. Manual Controls. The furnace operator sets the particular time and temperature, and he/she needs to check the top, bottom, left, and right temperature levels. This process takes a minimum of 6 hours to maximum of 2 days to 


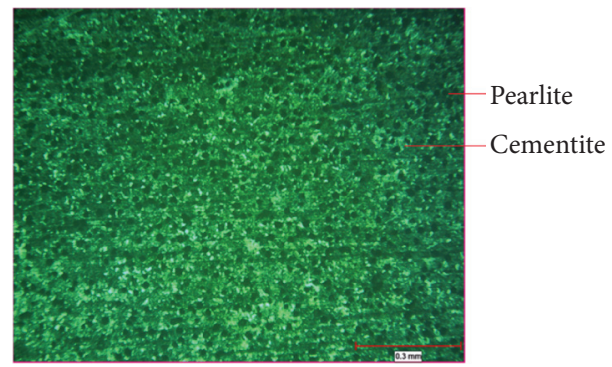

Figure 2: After heat treatment microstructure.

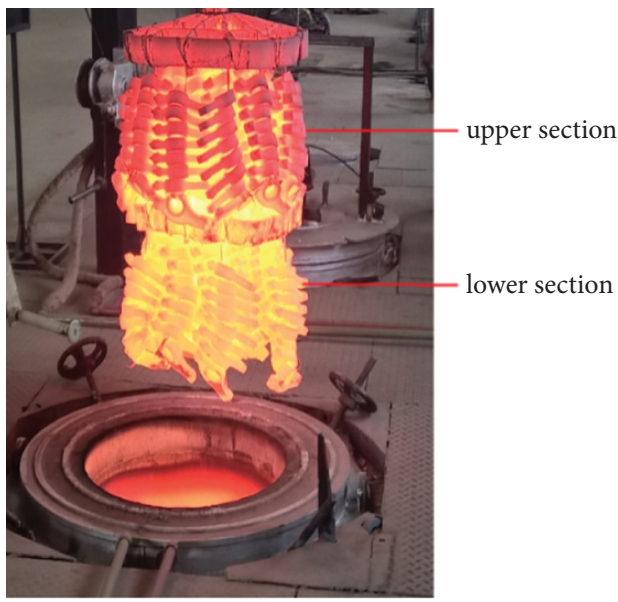

FIGURE 3: The undesired temperature variation in metal casting (difference in upper and lower section).

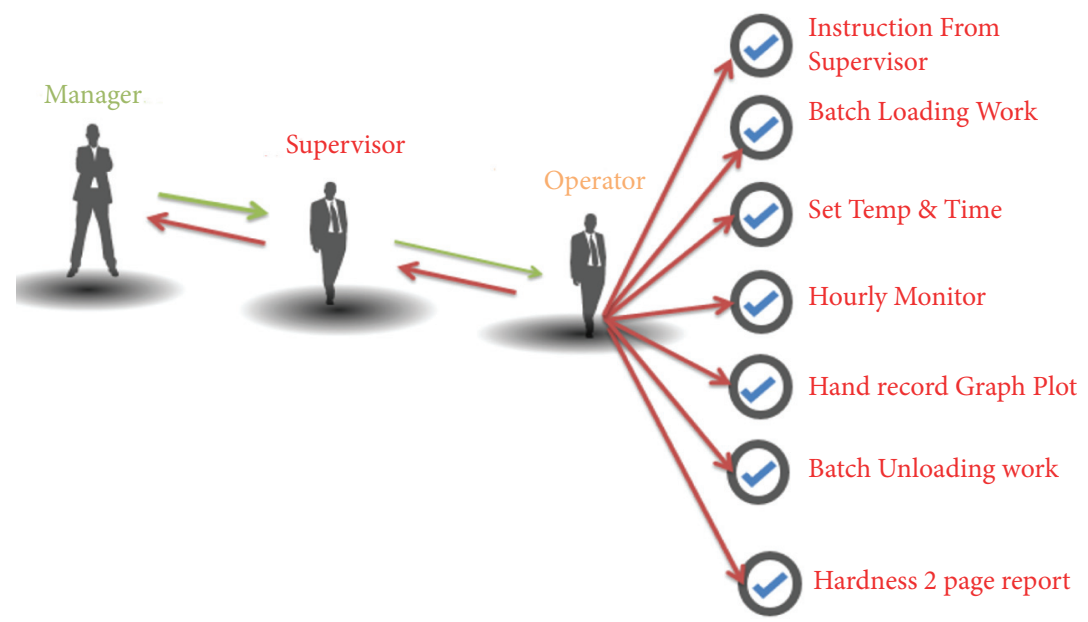

FIgURE 4: Manual instruction process flow.

complete. If any mistakes occur in this process, it consumes 250 units to 500 units per day; also, currently running parts of microstructure get uneven.

3.4. Analogy Measurement. In the previous method, the graph recorder of the heat treatment is in an analog format; the analog graph recorder automatically reads the four temperatures and plots the graph. For this method, the graph record uses more paper to plot and the hot environment damage; moreover, the recorder gets struck; if any mistake occurs in the previous completed record, we cannot analyze or check that in the paper form; the operator will be able to create the fake record if he has made any mistake in the process [5]. At the yearly, once auditing time the heat treatment area audit is more complicated, it contains a whole one-year graph record in the paper format. 


\section{Hardware Stability Analysis}

In order to design a reliable industrial automation system, the evaluation process at two stage architecture is used. The study required a simple microcontroller to carry out this operation. Hence, in this paper, at the first stage, we used the PIC16f877A controller shown in Figure 5, and at the second stage, we had an ARM-based 32 bit lpc2148 Philips microcontroller shown in Figure 6. The architecture used for evaluation performed well for three weeks, after which the system stopped functioning due its inability to withstand the high-temperature foundry environment [6-8]. Hence, it is inferred that, for HTP, a reliable microcontroller which can handle high temperature has to be used.

The furnace located in different parts of the unit has to be interconnected using local area network (LAN) so that a stable network is established for intermachine communication.

The above requirement is satisfied by an IoT-based Xmc4700 ARM Cortex M4 industry standard microcontroller which is resistant to high temperature and enables to safeguard the furnace from hazards due to explosion and fire. With the support of interfacing with automatic burner control and gas fired controlled dryer, the controller can operate in the furnace ambient temperature range upto $125^{\circ} \mathrm{C}$. Any compromise in maintaining a constant temperature during HTP can lead to severe consequence including wastage of all the preliminary casting process and the power consumed. The undesired variation in temperature during HTP is presented by the temperature-monitoring graph, as shown in Figure 7 . The respective real-time metal casting in a foundry is given in Figure 7. Hence, maintaining constant temperature is very primary for manufacturing durable accessories for automobile.

\section{Experimental Setup for Data Communication Using IoT}

5.1. Device and Furnace. The temperature measurement at the top, bottom, left, and right of the furnace is measured using four different temperature controllers interfaced with IoT-based Xmc4700 [9]. The PCB architecture which is based on IoT for the heat treatment process is shown in Figure 8 . While the flowchart for heat treatment process is shown in Figure 9.

The data are collected from Xmc4700 using RS485 Modbus communication to monitor the holding time of temperature and its set point [10]. The Modbus protocol reads temperature from Xmc IoT board at a baud rate of 9600 such that the temperatures from four corners of the furnace are collected within 800 millisecond. The study records the threshold temperature limit as $125^{\circ} \mathrm{C}$ and upon which if the temperature increases, the time gets restarted and the process is iterated in similar manner until the decision is made. The RS485 serial communication employs balanced data transmission and performs good noise rejection so that it can drive for long communication lines at relatively high data rates $[11,8]$.

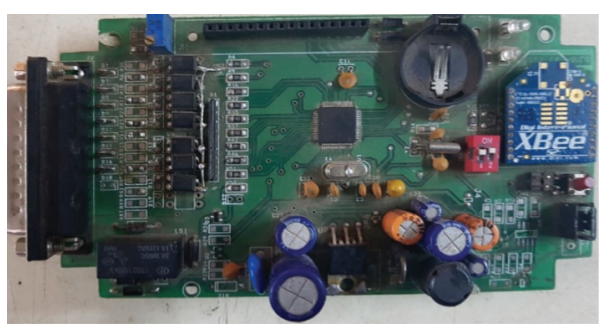

Figure 5: Hardware of PIC16f877a with Zigbee.

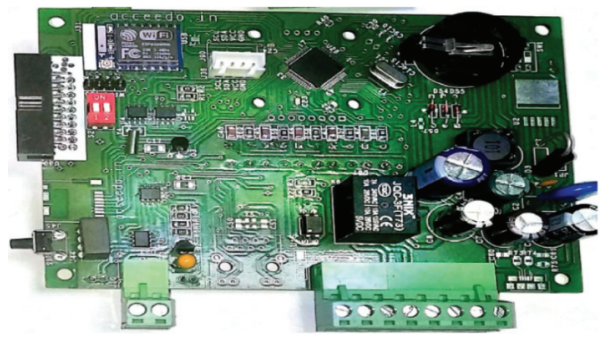

FIgURE 6: Hardware of lpc2148 with Wi-Fi.

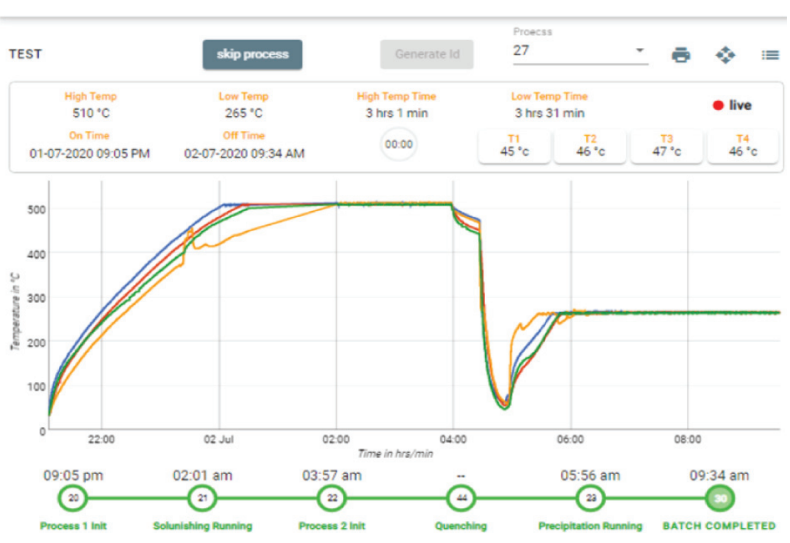

FIgURE 7: Temperature-monitoring graph.

5.2. Device and Server System. The XMC board collects the temperature information and transfers it through ESP8266 $\mathrm{Wi}$-Fi using hypertext transfer protocol (HTTP). It is a request/response protocol based on client/server-based architecture. The XMC collects the temperature data every 7 seconds and transfers it to the server through ESP8266 WiFi. The block diagram for machine monitoring and controlling is shown in Figure 10. The server collects the furnace temperature data to store it in a database. It gives an acknowledgment with temperature setpoint and performs data analysis to predict warnings and intimates the same through an e-mail notification.

Digital Application virtual Engineering (DAvE) is a $\mathrm{C} / \mathrm{C}++$ code development platform which can generate code for microcontroller and software driver applications [12]. It is a free and unlicensed application environment that can automatically create user-defined functions using C-level templates. DAvE generates code to read temperature using RS485 from the temperature controller and store the data in EPROM AT24C64 ATMEL. The microcontroller collects the 

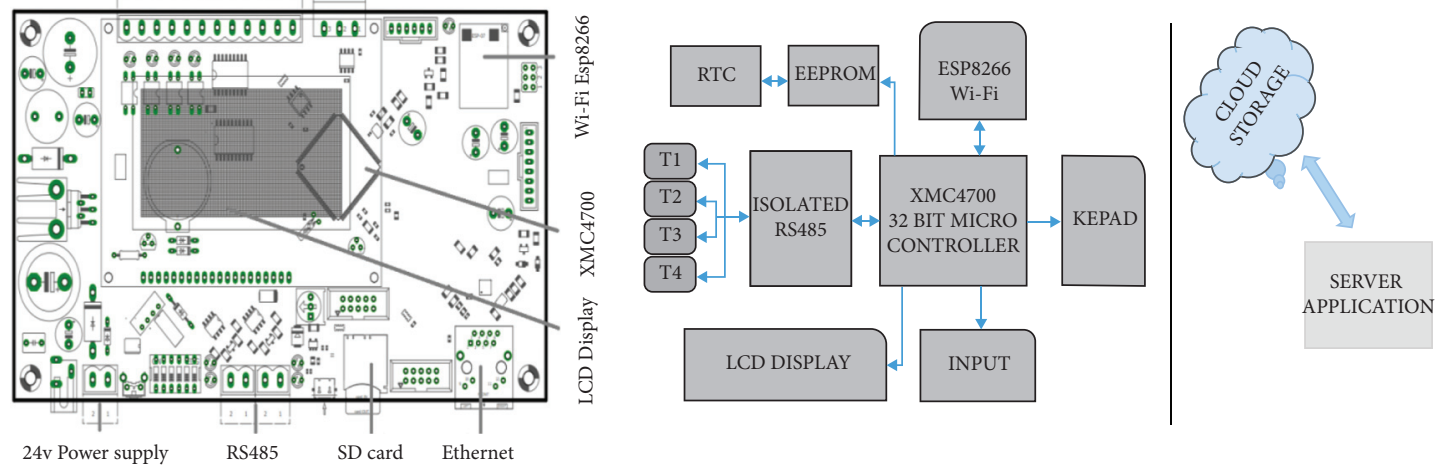

FIgURE 8: IoT-based PCB architecture for the heat treatment process.

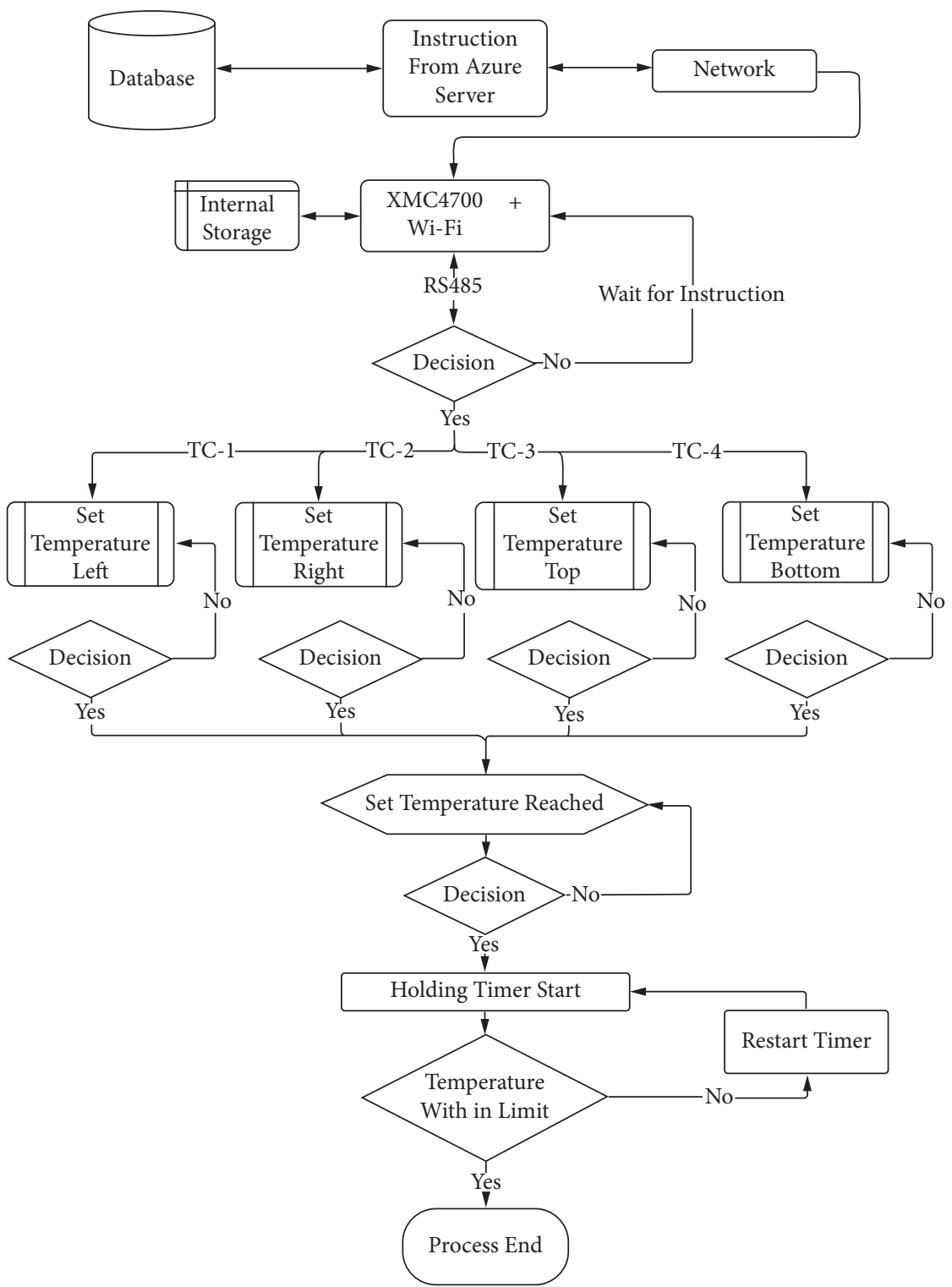

Figure 9: Flow diagram for heat treatment process. 


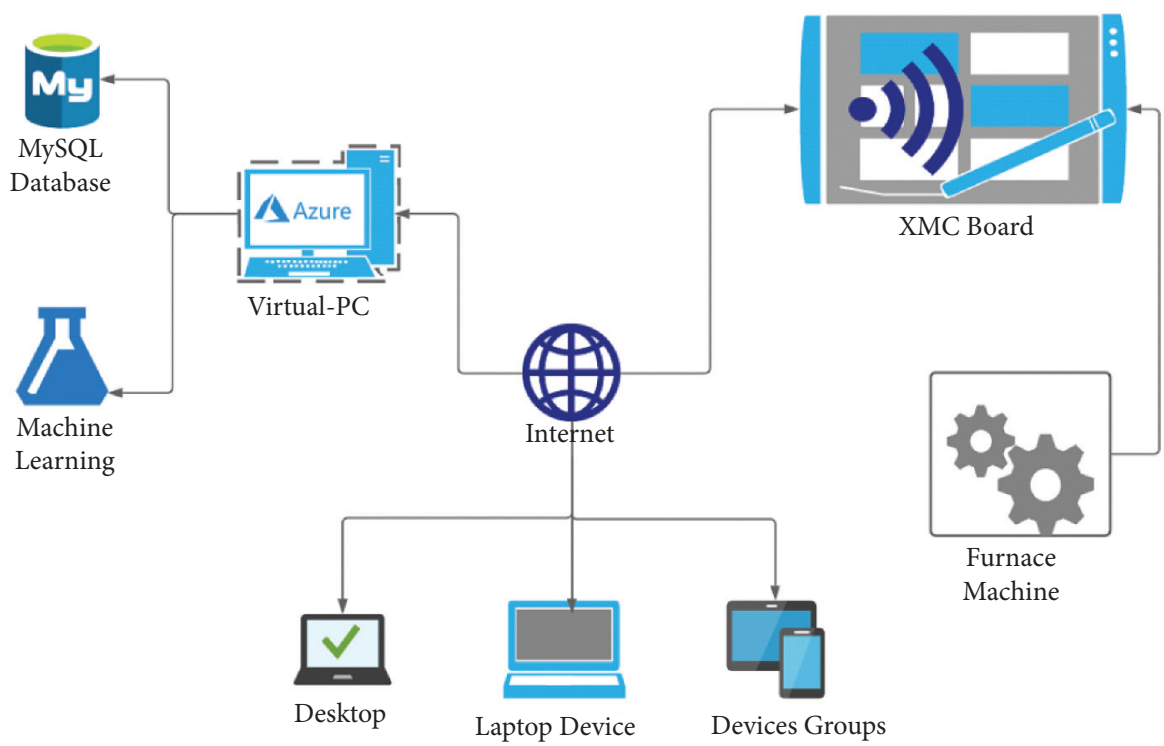

Figure 10: Block diagram for machine monitoring and control.
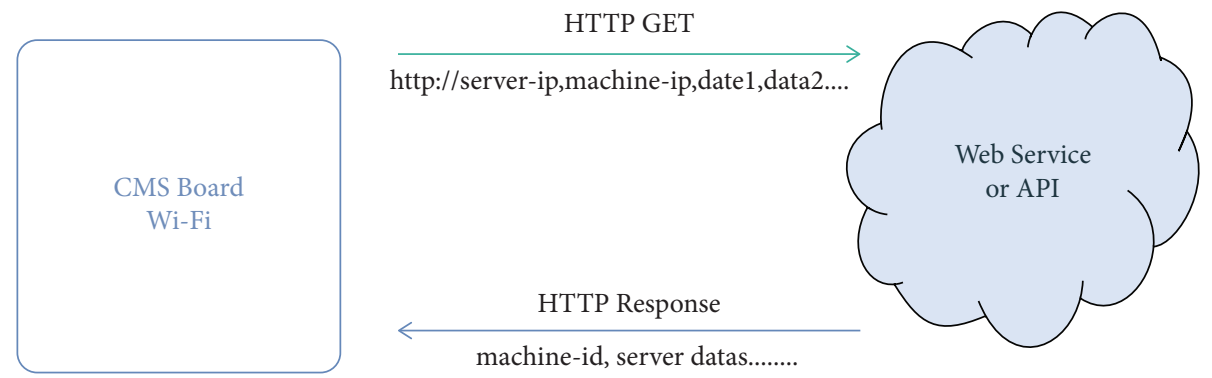

FIgURE 11: Data communication by HTTP request and response.

The steps and flow of proposed are summarized below.

Step 1: initialize temperature $\left(160^{\circ} \mathrm{C}\right)$ and time setting instruction from Azure Server

Step 2: XMC board receive initial parameter through and save in internal memory

Step 3: temperature set point received through XMC board and assigning to four temperature controller (TC-1, TC-2, TC-3, and TC-

4) through RS485 communication

Step 4: wait until temperature reaches set point

Step 5: once set temperature reached the XMC board, start the holding temperature time

Step 6: XMC board monitor and communicate the temperature to azure cloud

Step 7: if temperature deviation on hold time, the control restarts the time and timer will start again

Algorithm 1: Heat treatment process flow.

temperature data from EPROM and transfers it to Microsoft Azure cloud using Wi-Fi module ESP8266, as shown in Figure 11. (Algorithm 1)

\section{Result and Discussion}

This proposed IoT-based heat treatment prototype is an alternative for analog graph recorder and manual control process $[13,14]$. Figure 12 shows how the industrial automation model works in heat treatment process. This system designs with high industrial standard which can withstand in a high-temperature area. This system used Wi-Fi to transfer the temperature data to the system application, and moreover, this system can monitor the furnace temperature continually as reported in Figure 13. 

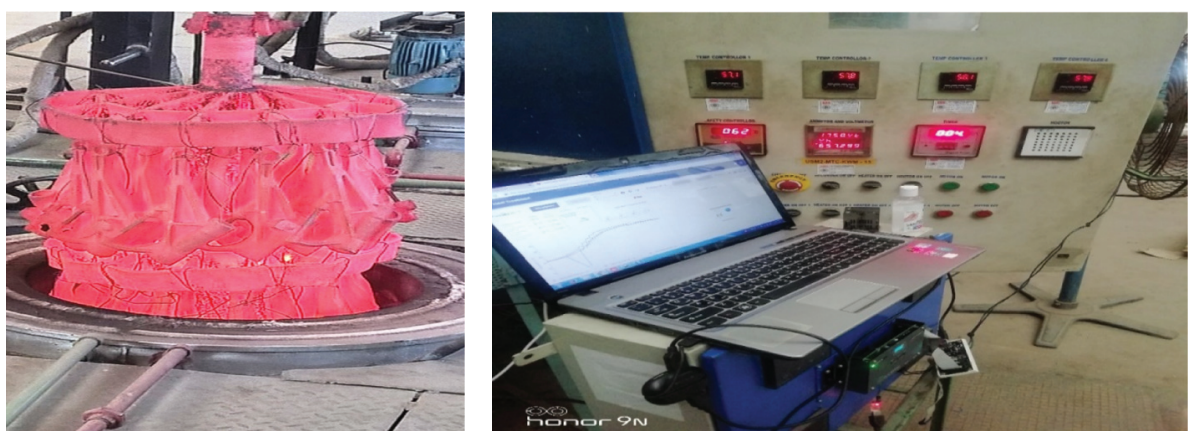

FIGURE 12: Industrial automation model of the heat treatment process.

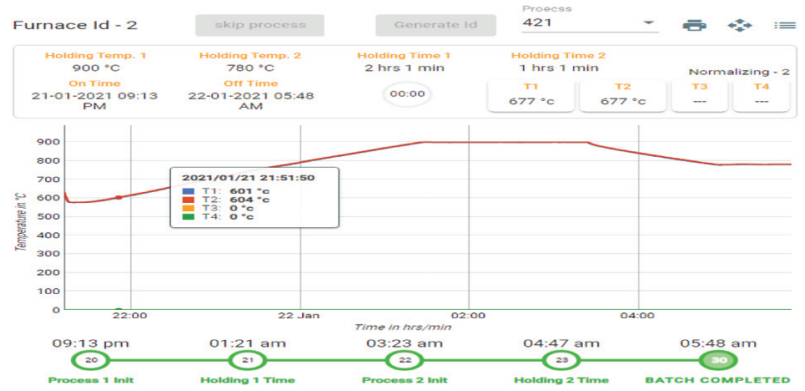

Figure 13: Browser http request and response chart.

\section{Conclusion}

To verify the accuracy of the proposed system, we conducted experiments in three cases. In the first case, we examined the microcontroller stability working in the foundry environment. In the second case, we examined the communication between the board and the furnace temperature controller. In the third case, we examined communication between board to cloud server application that controls and monitors the entire furnace; finally, we merge the three experiments; this proposed system helps to plot the live furnace temperature graph, deduct the furnace temperature failure at an earlier stage, reduce the power consumption, increase the product quality, and finally proved industrial 4.0 can be applied for foundry. The study focuses entirely on the temperature sensing, monitoring, and controlling it; however, the other parameters defining the manufacturing process are not taken into account. From the above results, it can be seen that the proposed method can be effectively utilized in maintaining the furnace temperature based on the requirements. It can hence be utilized on other temperature sensing applications to maintain the optimal temperature in sensor applications.

\section{Data Availability}

The datasets used and/or analyzed during the current study are available from the corresponding author on reasonable request.

\section{Conflicts of Interest}

The authors declare that there are no conflicts of interest.

\section{References}

[1] J. Wan, H. Cai, and K. Zhou, "Industrie 4.0: enablingtechnologies," in Proceedings of the Intelligent Computing and Internet of Things (ICIT), 2014 International Conference on. IEEE, pp. 135-140, Harbin, China, January 2015.

[2] T. M. ZeeshanKaleem and C. Lee, "Energy efficient outdoor light monitoring and control architecture using embedded system," IEEE Embedded systems letters, vol. 8, no. No.1, 2016.

[3] T. Andreas and F. O. luis, "Voltage control in PV-rich LV networks without remote monitoring," IEEE Transaction on Power systems, vol. 32, no. .2, 2017.

[4] M. Fazio and A. Puliafito, "Cloud4sens: a cloud-based architecture for sensor controlling and monitoring," IEEE Communications Magazine, vol. 53, no. 3, pp. 41-47, 2015.

[5] W. Dai, "Redesign distributed PLC control system using IEC 61499 function blocks," IEEE Transactions on Automation Science and Engineering, vol. 9, no. 2, 2012.

[6] C.-H. Lu and C. C. Tsai, "Adaptive decoupling predictive temperature control for an extrusion barrel in a plastic injection molding process," IEEE Transactions on Industrial Electronics, vol. 48, no. 5, pp. 968-975, 2001.

[7] E. Vuerich, F. Malaspina, M. Barazutti, T. Georgiadis, T. Georgiadis, and M. Nardino, "Indoor measurements of microclimate variables and ozone in the church of San Vincenzo (Monastery of Bassano Romano - Italy): a pilot study," Microchemical Journal, vol. 88, no. 2, pp. 218-223, 2008.

[8] G. Visco, S. H. Plattner, P. Fortini, and M. Sammartino, "A multivariate approach for a comparison of big data matrices. Case study: thermo-hygrometric monitoring inside the CarcerTullianum (Rome) in the absence and in the presence of visitors," Environmental Science and Pollution Research, vol. 24, pp. 1-15, 2017.

[9] T. Liu, K. Yao, and F. Gao, "Identification and autotuning of temperature-control system with application to injection molding," IEEE Transactions on Control Systems Technology, vol. 17, no. 6, pp. 1282-1294, 2009.

[10] C.-C. Tsai and C.-H. Lu, "Multivariable self-tuning temperature control for plastic injection molding process," IEEE Transactions on Industry Applications, vol. 34, no. 2, pp. 310-318, 1998 .

[11] G.-Y. Liao, Y.-J. Chen, W.-C. Lu, and T.-C. Cheng, “Toward authenticating the master in the Modbus protocol," IEEE Transactions on Power Delivery, vol. 23, no. 4, pp. 2628-2629, 2008.

[12] T. Cucinotta, A. Mancina, G. F. Anastasi et al., "A real-time service-oriented architecture for industrial automation," IEEE 
Transactions on Industrial Informatics, vol. 5, no. 3, pp. 267-277, 2009.

[13] Y. Tabunschikov and M. Brodatch, "Indoor air climate requirements for Russian churches and cathedrals," Indoor Air, vol. 14, no. s7, pp. 168-174, 2004.

[14] F.-J. Garca-Diego and M. Zarzo, "Microclimate monitoring by multivariate statistical control: the renaissance frescoes of the Cathedral of Valencia (Spain)," Journal of Cultural Heritage, vol. 11, pp. 339-344, 2010. 